\title{
Cutaneous Lupus Erythematosus
}

\author{
Allison Chabassol \\ Class of 2013, Faculty of Medicine, Dalhousie University
}

Lupus erythematosus (LE) is a heterogeneous group of chronic autoimmune inflammatory diseases, ranging from solely cutaneous symptoms to systemic disease with extensive visceral involvement. ${ }^{1}$ Lupus was first named in the Middle Ages, but evidence suggests that Hippocrates described it long before that time. ${ }^{2}$ Its intricate pathogenesis makes lupus a mainstay in the world of dermatological research, especially since roughly $75 \%$ of affected individuals experience cutaneous symptoms at some point during the course of the disease. ${ }^{3}$ As a result, thorough knowledge of cutaneous lupus erythematosus (CLE) is integral to successful management of affected patients.

\section{Epidemiology of LE}

The prevalence of systemic lupus erythematosus (SLE) is 17-48 per 100,000 worldwide. ${ }^{3-4}$ Although it was once believed that CLE was 2-3 times more common than SLE, ${ }^{5}$ emerging evidence suggests that the incidence rates are comparable. Typically, patients with LE are between the ages of 20-40 with a female predominance that varies according to subtype. ${ }^{1}$ Patients with African ancestry have been found to be slightly more susceptible. ${ }^{1}$

As previously mentioned, cutaneous manifestations are one of the most common clinical complaints of patients with LE, and are the second most common presenting symptom after joint involvement. ${ }^{3}$ Although CLE is rarely life-threatening, it contributes disproportionately to disease burden through vocational disability and effects on personal and social well-being, in addition to significant medical and social costs. ${ }^{7}$

Although CLE typically occurs in conjunction with systemic involvement, it is important to clarify that it may exist independently of extracutaneous symptoms. ${ }^{3}$ This distinction is important - it is crucial to identify the specific subtype of CLE, since it functions as an indicator of the extent of systemic disease, in addition to directing the management plan and affecting the patient's prognosis. ${ }^{8}$

\section{Pathogenesis of CLE}

The pathogenesis of CLE is complicated and has not yet been fully elucidated. The mechanisms involved in the development of the disease continue to be an area of active investigation.

Patients with CLE have dysregulated apoptosis, as well as decreased apoptotic cell clearance. Research shows that UV light induces apoptosis of keratinocytes through IL-1 \& TNF- $\alpha$, and occasionally, at very high doses, leads to necrosis. ${ }^{9-10}$ There is also increased antibody binding to keratinocytes following UV exposure, which could be a result of UV-induced translocation of antigens to the cell surface or UV-induced alteration of antigen properties. UV light induces apoptosis of keratinocytes, and apoptotic blebs may be presented to lymphocytes, resulting in the stimulation of an immune response. ${ }^{9-10}$

It is believed that patients with CLE have upregulated interferon- $\alpha$ inducible genes. ${ }^{9-10}$ Local production of type 1 interferons leads to recruitment of chemokines and $\mathrm{T}$ lymphocytes to the skin, which results in Th1-biased inflammation. It has been thought that B cells may not be as important in the pathogenesis of CLE, compared to SLE, since anti-B cell therapy is not a particularly effective treatment of CLE. ${ }^{9-10}$

\section{Classification: Clinical, Histopathological \& Immunological Diagnostic Features}

The classification of CLE consists of a tiered system of divisions based on histopathologic examination and cutaneous lesion morphology. CLE is first classified as either LE-specific or LE-nonspecific. LE-specific cutaneous manifestations are further classified as chronic (CCLE), subacute (SCLE) or acute (ACLE), each of which consists of a variety of different subtypes. ${ }^{8}$ In a prospective study completed in a tertiary care dermatological reference center, the frequency distribution for CLE was found to be: $67.5 \%$ for Discoid LE (the most common form of CCLE), 18.4\% for SCLE, 6.1\% for ACLE and 6.1\% for LE-nonspecific CLE. ${ }^{11}$ However, it is not uncommon to have difficulty identifying a single subtype of CLE since overlapping features are prominent on the disease continuum. ${ }^{12}$ In patients with SLE, the presence of more than one LE-specific subtype is especially prevalent. ${ }^{1}$ 
LE is regarded as the great imitator, so confidence in classification is essential for prompt and proper diagnosis and effective management, which varies according to subtype.

\section{LE-Nonspecific CLE versus LE-Specific CLE}

LE-nonspecific CLE and LE-specific CLE are distinguished on the basis of histopathological characteristics described originally by the Gilliam and Sontheimer classification of LE-associated skin lesions. It is based on the presence of interface dermatitis-inflammation at the basal membrane zone of the interfollicular epidermis. ${ }^{7}$ The following hallmarks of lichenoid tissue reaction are common amongst LE-specific cutaneous lesions: hyperkeratosis; epidermal atrophy; liquefactive degeneration of the epidermal basal-cell layer; a mononuclear cell infiltrate focused at the dermo-epidermal junction, perivascular areas and perifollicular areas; thickening of the basal membrane; and melanin pigment incontinence. ${ }^{12}$ In contrast, LE-nonspecific lesions may be seen as part of another disease process - their histology is not distinct for LE. ${ }^{4}$

\section{LE-Nonspecific Lesions}

LE-nonspecific lesions are extremely prevalent in patients with SLE and, therefore, manifestations such as oral ulcers and photosensitivity are included as part of the ACR diagnostic criteria for SLE. ${ }^{1}$ Furthermore, the presence of LE-nonspecific lesions may be used as an indicator of underlying SLE activity. Research has revealed that the presence of such lesions is associated with higher activity scores than the presence of only LE-specific lesions or a combination of both types of lesions. ${ }^{1,4,13}$ Likewise, the number of different skin lesion types has also been found to correlate with disease activity. Patients with three or more different lesion types (specific or nonspecific) were found to have higher disease activity scores. ${ }^{13}$ Photosensitivity in particular is a markedly sensitive indicator of SLE. It is estimated that $50-93 \%$ of SLE patients experience photosensitivity. ${ }^{1}$

Generally speaking, LE nonspecific lesions may be divided into three main groups: cutaneous vascular disease, non-scarring alopecia, and other dermatologic conditions.

Cutaneous vascular conditions are often indicators of underlying systemic vascular pathology. As a result, recognition of such conditions in patients with SLE is extremely important. Raynaud's phenomenon is the most common vascular reaction in patients with SLE, occurring in an estimated $40 \%$ of affected patients. ${ }^{14}$
The presence of this phenomenon is associated with higher disease activity scores and poorer prognosis. ${ }^{15-16}$

LE-nonspecific vasculopathy occurs generally as a vasculitis in patients with SLE as a result of antiphospholipid antibodies. In such cases, it is important to investigate the possibility of vasculopathy due to thromboembolism, since clinical findings would be similar. ${ }^{1}$

Urticaria is also not uncommon in patients with SLE and is speculated to be a result of immune dysregulation. In one study, it was found that 44-73\% of SLE patients had chronic urticaria. ${ }^{17}$

Other LE-nonspecific cutaneous conditions include: cutaneous vascular disease (periungal telangiectasia, livedo reticularis, thromophelitis, erythromelalgia), non-scarring alopecia ("lupus hair," telogen effluvium, alopecia areata), and other dermatologic conditions (sclerodactyly, rheumatoid nodules, calcinosis cuti, LE-nonspecific bullous lesions, papulonodular mucinosis, cutis laxa/anetoderma, acanthosis nigricans, erythema multiforme, leg ulcers, lichen planus). ${ }^{1}$

\section{LE-Specific Lesions \\ ACLE}

Important Points:

ACLE occurs almost exclusively in patients with SLE. Patients with ACLE have almost $100 \%$ chance of having clinically significant systemic manifestations. ${ }^{7}$ Photosensitive distribution with severity that flucates with sun exposure and SLE severity. ${ }^{8}$ Post-inflammatory pigmentary changes may occur, but scarring is rare. ${ }^{1}$

\section{Clinical Presentation:}

LOCALIZED: Classic "butterfly" rash - confluent, erythematous, edematous rash located on the nasal ridge and malar eminences. May extend to forehead, chin or V-area of the neck. Spares the nasolabial folds. ${ }^{1}$ GENERALIZED: Pruritic, maculopapular rash typically located on extensor aspects of the arms and hands but sparing the knuckles. ${ }^{1,8}$

\section{Associated Findings:}

Diffuse hair thinning, receding hairline, telangectasias and erythema of the proximal nail fold., ${ }^{3,7}$

\section{Histological Findings:}

Interface dermatitis with basal layer vacuolization, edema in the upper dermis, focal liquefactive degeneration or the basal cell layer and lymphocytic dermal infiltrates. ${ }^{7,8,18}$ 
Immunological Findings:

Lupus band test identifies granular deposits of immunoglobulin and complement at the dermoepidermal junction. It is useful for distinguishing between SLE and CLE, because it is positive for both affected and unaffected areas in SLE but only in affected areas in CLE. Other conditions, as well as healthy sun exposed skin, may produce positive results.,19

\section{Serological Findings:}

Typically ANA and anti-dsDNA positive and have low complement. Anti-Sm antibodies have a strong specificity for SLE and are used to determine underlying systemic disease. ${ }^{18}$

\section{SCLE}

Important Points:

Associated with mild systemic symptoms, most commonly musculoskeletal. ${ }^{20}$ Lesions are traumainduced and photo-aggravated. Many drugs have also been linked to the onset and exacerbation of symptoms. ${ }^{20}$ SCLE commonly heals without scarring or dermal atrophy, however telangectasias and post-inflammatory hypopigmentation are common. ${ }^{8}$ Papulosquamous type, leukopenia and high titers of ANA and anti-dsDNA antibodies have all been linked to increased risk of developing SLE. ${ }^{1}$

\section{Clinical Presentation:}

Characterized by recurring, non-scarring symmetrical lesions occurring in a photosensitive distribution. Lesions begin as erythematous macules and/or papules and progress to hyperkeratotic plaques. ${ }^{1,8,20}$

\section{Histological Findings:}

Compared to ACLE, SCLE has more prominent vacuolization on the basal layer, marked atrophy of the epidermal/adnexal epithelium and denser lymphocytic infiltrate. ${ }^{7}$ Compared to DLE, SCLE tends to have less hyperkeratosis, follicular plugging, adnexal infiltrates and dermal melanophages. ${ }^{20}$

Immunological Findings:

Often characterized by a pattern of "dust-like" particles of IgG that are visible upon direct immunofluorescence and are associated with the presence of Ro/SSA autoantibodies. Unique to SCLE, but low diagnostic sensitivity. ${ }^{3,7}$

\section{Serological Findings:}

Anti-Ro/SSA is most important and is found in 70-90\% of cases. Anti-La/SSB is found in $35-70 \%$ of cases. Transplacental passage of anti-Ro and/or anti-La antibodies can react with fetal antigens and result in neonatal lupus erythematosus. ${ }^{7}$

\section{CCLE}

Important Points:

Occurs in patients with long term, low-grade illness. Discoid type is most common. ${ }^{3,8}$ Classified as localized, (70\%) or generalized (30\%). ${ }^{8}$ Localized lesions typically occur above the neck, whereas generalized lesions occur above and below the neck..$^{1,20}$ Trauma induced and exacerbated by sun, but less so than ACLE and SCLE. ${ }^{1,8}$ Generally resolves with atrophy, irreversible scarring, pigmentary changes, telangectasias and permanent-scarring alopecia. ${ }^{1,7}$

\section{Clinical Presentation:}

One or more clearly demarcated, erythematous, disc-shaped papules or plaques with adherent hyperkeratotic scale extending into surrounding pilosebaceous follicles (follicular plugging). Removal of the scale is quite painful and may result in the "carpet tack sign." ${ }^{1,7}$ Nail findings may include nail plate dystrophy, pitting, ridging, leukonychia striata, onycholysis, clubbing, nail bed erythema and telangectasias. ${ }^{1,7,20}$

Histological Findings:

Hyperkeratosis of the epidermis, keratotic follicular plugging, vacuolar degeneration of basal keratinocytes and dermal mononuclear cellular infiltrate (infiltrate extends deeper than in ACLE and SCLE), ${ }^{8,20}$

\section{Immunological Findings:}

Direct immunofluorescence reveals immunoglobulins and complement in a granular band along the dermoepidermal junction..$^{7-8}$ Lupus band test is positive in $90 \%$ when performed on lesional skin and in $10-20 \%$ when performed on non-lesional skin. ${ }^{8}$

\section{Serological Findings:}

Only $1 / 3$ of patients are ANA positive. Positive serology tends to be more common in patients with generalized disease, those who have had DLE for a long time and elderly patients. Serology should be monitored to track disease progression. ${ }^{7-8}$

\section{Treatment of CLE and Associated Adverse Effects}

Although CLE has several different forms, treatment regimens remain similar across the board, with the goal to prevent lesion progression and enhance patient appearance. ${ }^{3}$

Initial management focuses on patient education. It is essential to identify provocative agents and develop strategies to deal with these precipitating factors. Heat, 
certain drugs, and most importantly, sunlight should all be avoided. Sunscreen and sun avoidance is the cornerstone of CLE treatment. ${ }^{21}$ It is recommended that patients photo-protect, both physically, through tightly woven clothing and hats and chemically, through sunscreens of at least SPF 30 with both UVB and UVA protection. ${ }^{20}$ This is especially important in patients with SCLE, since $64 \%$ will develop skin lesions after one week of irradiation. However, it is not to be neglected in other patients, considering many who report no photosensitivity actually produce abnormal photoprovocative test results, which is likely accounted for by a delayed reaction following sun exposure. ${ }^{21}$

Initial therapy often also includes a medium potency topical corticosteroid, applied daily to lesional skin. ${ }^{20}$ High potency steroids may be used, but are typically reserved for thicker skin. Once improvement occurs, patients are instructed to taper corticosteroid potency. ${ }^{21}$ Generally, however, topical corticosteroids alone do not result in adequate improvement. For localized lesions, intralesional corticosteroid injections are an option. However, side effects include subcutaneous atrophy and leukoderma at the site of injection..$^{20}$ Oral corticosteroid therapy is avoided when possible, though can be useful in short courses while waiting for slow-acting agents to take effect. ${ }^{3,20}$ Nonetheless, it is important to note that LE patients are at increased risk of avascular necrosis and thromboembolism. ${ }^{21}$

Antimalarial agents have been used for decades as treatment for LE and continue to play a major role in successful treatment of these conditions. Roughly $75 \%$ of patients with SCLE will respond to mono or combination antimalarial therapy. ${ }^{20}$ Hydroxychloroquine, chloroquine and quinacrine are all used in practice, ${ }^{21}$ with the former two being used most frequently due to less serious adverse effects. ${ }^{22}$

Antimalarials have more than one mechanism of action. They increase vacuolar $\mathrm{pH}$, which decreases the immune response to autoantigens due to alteration in antigen processing and presentation. However, they also inhibit the release of pro-inflammatory cytokines. In addition, there is evidence that antimalarials inhibit granulocyte migration and phospholipase A2 activity, which may be implicated in their effectiveness in treating LE. ${ }^{21}$

Typically, patients are started on 200mg once daily of hydroxychloroquine to determine gastrointestinal tolerance and, in the absence of any issues, the dose is increased to $200 \mathrm{mg}$ twice daily. ${ }^{21-22}$ It often takes roughly two months for effects to become apparent. ${ }^{22}$
If monotherapy proves unsuccessful, quinacrine $100 \mathrm{mg}$ once daily may be added to the regimen. It has been found that hydroxychloroquine and quinacrine combination therapy is effective in treating patients for which hydroxychloroquine monotherapy has proved ineffective. If combination therapy continues to be ineffective after approximately six weeks, hydroxychloroquine may be replaced with chloroquine in a dose of $250-500 \mathrm{mg}$ once daily. ${ }^{20-21}$ However, hydroxychloroquine and chloroquine should never be used in combination due to the increased risk of retinopathy. ${ }^{3}$ It is recommended that patients remain on antimalarial therapy for one to two years to fully suppress cutaneous LE activity. ${ }^{20}$

In addition to being an effective treatment for LE, antimalarial therapy has a variety of other benefits including improvement of fatigue, headache, fever, arthralgias, arthritis, pleuritis, and pericardial inflammation. ${ }^{3,21}$ In addition to these benefits, hydroxychloroquine has antithrombotic effects and will also lower cholesterol.,21 However, like any treatment, antimalarials are not without adverse effects; fortunately, side effects generally occur in less than $10 \%$ of patients. ${ }^{23}$ Gastrointestinal complaints are the most common adverse event for all types of antimalarials, but are typically evanescent, and can be ameliorated by decreasing drug dose. ${ }^{22}$ The most serious complication of hydroxychloroquine/ chloroquine treatment is irreversible retinal toxicity. Patients taking this therapy should be followed closely by an ophthalmologist - every six months for hydroxychloroquine and every four months for chloroquine.,22 Other less common symptoms associated with hydroxychloroquine/chloroquine include blue-grey hyperpigmentation, urticaria, MSK flu-like symptoms, headache, nervousness, insomnia, psychosis, and seizures. Hematologic and hepatic effects have also been reported, but are exceptionally rare. There are also several case reports of cholorquine being associated with cardiac conduction issues, which should be considered in patients with a pre-existing conduction defect. ${ }^{24}$ In patients taking quinacrine, adverse effects include GI symptoms, discoloration of the skin and bodily secretions, eczematous skin lesions, exfoliative skin lesions, headache, and dizziness. Retinal toxicity is not an issue. ${ }^{3,21}$

Although the mechanism is unclear, there is evidence to suggest that smoking interferes with the effectiveness of antimalarials. One study of patients with DLE and patients with SCLE found that cutaneous lesions were responsive to antimalarials in $91 \%$ of non-smoking patients, but only $40 \%$ of smokers. ${ }^{25}$ Furthermore, it has 
been suggested that smoking also directly exacerbates cutaneous LE lesions. ${ }^{20}$ Therefore, smoking cessation plays a key role in successful management of LE through antimalarial treatment.

Another issue that arises with the use of antimalarials is safety in pregnancy. Initially, withdrawal of antimalarial therapy was recommended in pregnant patients with LE. However, since hydroxychloroquine and chloroquine have a high affinity for binding in tissue, it takes several months for them to be cleared from the system. As a result, stopping therapy at the first sign of pregnancy does not eliminate fetal drug exposure. ${ }^{21}$ Evidence has since indicated that antimalarials have a good safety profile and prove quite useful in suppressing LE flares that may occur during pregnancy. ${ }^{26-28}$ One study revealed lower frequency of LE flares during pregnancy, as well as absence of teratogenic effects in children after a three-year follow-up. ${ }^{27}$ Generally, hydoxychloroquine is considered safer than chloroquine since it is less toxic, binds less readily to tissues, and is less able to cross the placenta. ${ }^{27}$ Safety of these drugs in breastfeeding mothers has not yet been established..$^{22}$

Dapsone and retinoids, although less effective than antimalarials, are also a treatment option for patients with CLE. Dapsone has been found to successfully treat patients with bullous LE, SCLE and DLE. ${ }^{29-31}$ It is also a good choice for patients with LE who also have vasculitis. Retinoids have proved useful in the treatment of hypertrophic DLE. ${ }^{32}$

Thalidomide has been well publicized for its teratogenic effects; however, despite its generally bad reputation it is extremely effective in the treatment of CLE. Research shows that on average, thalidomide produces therapeutic benefit in $90 \%$ of patients with CLE. ${ }^{33}$ The therapeutic effects of thalidomide are thought to be a result of decreased TNF- $\alpha$ activity and inhibited angiogenesis. ${ }^{3}$ Careful patient selection and vigilant drug monitoring are crucial when using thalidomide therapy. Patients should be extensively counseled about the drug. Female patients of childbearing age are required to use two effective forms of contraception one month prior to starting therapy, during therapy, and one month after the completion of therapy. ${ }^{33}$ In terms of potential adverse effects, the most commonly reported are mild, and include constipation, weight gain and sedation. ${ }^{3}$ Thalidomide-induced peripheral neuropathy is a significant concern as it has been reported to occur in up to $50 \%$ of patients in some series and is potentially irreversible. Therefore, neurological testing of patients on thalidomide is mandatory. Other less common but serious effects include teratogenicity, ovarian failure, and thrombosis. ${ }^{33}$

Refractory CLE is defined as a disease that does not respond to systemic antimalarial and corticosteroid therapy added to topical therapy, and with which concomitant pathogenic processes have been excluded. ${ }^{34}$ First, when assessing refractory disease, consideration should be given to modifiable factors such as sun-exposure, smoking and drugs that are known to exacerbate CLE. Although there are no universal guidelines for the treatment of refractory CLE, some medications have been identified as more effective than others. Thalidomide and methotrexate have shown impressive results with patient response rates of over $90 \%$. $^{34}$

\section{Prognosis and Quality of Life}

An outcome measurement for CLE has recently been developed in the form of CLASI (Cutaneous Lupus erythematosus disease Area and Severity Index). CLASI has two scores, one for disease activity and one for damage as a result of the disease. Activity is a summative score of erythema, scale/hypertrophy, mucous membrane involvement, acute hair loss or non-scarring alopecia. Damage is a summative score of dyspigmentation or scarring, including scarring alopecia..$^{35-36}$ The availability of such a scale, although not a perfect representation of such a multidimensional disease, can help quantify a patient's condition, which is helpful in clinical practice, and is useful for clinical trials. $^{36}$

Prognosis for patients with CLE is strongly connected to severity and extent of systemic involvement. Presently, prognosis is favourable, with 10-year survival exceeding $80 \%$. Most fatalities associated with LE occur as a result of systemic involvement, especially renal and CNS and infections. ${ }^{5}$ Therefore, this is of particular concern in patients with ACLE since it is almost exclusively associated with SLE.

Quality of life is a major concern in patients with dermatologic conditions. Psychiatric morbidity is higher in the dermatologic population than the general population - with prevalence ranges of $20-40 \%$ and $11-30 \%$, respectively. ${ }^{37}$ It is no surprise that patients with CLE are at risk for mental health issues as a result of poor quality of life associated with their illness. ${ }^{38}$ Researchers have shown that presence of cutaneous disease has a clear effect on patients' quality of life, and that this effect is most significant for those with generalized DLE. ${ }^{38}$ Evidence suggests that factors related to poor quality of life include female gender, young 
age, severe or generalized lesions, and distribution of lesions, particularly on the face. ${ }^{37}$ Mental health is crucial to overall health, and practitioners should be aware of the potential serious effects CLE can have on quality of life and patient well-being.

\section{Transition of Cutaneous LE into Systemic LE}

It is important for practitioners to recognize the potential for CLE to progress to more widespread systemic disease. ACLE is exclusively associated with SLE, thus this is applicable mainly to patients with SCLE and CCLE. It is difficult to predict disease course, however, a prospective multicenter study found that patients with signs of nephropathy, arthralgias and elevated ANA titers were at increased risk of developing extracutaneous LE symptoms, and that risk increased with the number of positive variables. ${ }^{39}$ Therefore, it may be beneficial to monitor such patients closely and tailor treatment accordingly. ${ }^{5}$ In terms of slowing the progression of CLE to SLE, research is limited. One study of military recruits, however, demonstrated an association between the use of antimalarial therapy and an increased lag time between first systemic symptom to fully developed SLE. ${ }^{38}$

Approximately $5-10 \%$ of patients with CDLEand $50-60 \%$ of patients with SCLE will develop extracutaneous involvement and progress to SLE during the course of their disease. ${ }^{5,40}$

\section{CLE and Malignancy}

Patients with LE are at an increased risk for the development of malignant neoplasms, particularly lymphoma and carcinoma of the skin. The frequency of malignancy in patients with SLE has been reported to be between 2.4 and $13.8 \%{ }^{41}$ Researchers are unsure of the exact mechanism. It has been speculated that malignant transformation may be linked to the disease process itself, or that it occurs as a result of immunosuppressive therapy that is often used in patients with LE. ${ }^{42}$ It has also been suggested, however, that antimalarials may serve as a protector against malignant transformation through mutation prevention, inhibition of telomerase, increased synthesis of p53 and improvement of DNA cell repair. ${ }^{42}$ Regardless, it is important to be aware of the possibility of neoplasm development when managing patients with CLE.

\section{Conclusion}

Accurate diagnosis of CLE is crucial, as it signifies the extent of systemic disease, directs the management plan, and indicates patient prognosis. CLE is the second most common presenting symptom and one of the most common patient complaints of individuals with
LE. It also contributes disproportionately to vocational disability. Therefore, regardless of the field of medicine, an awareness of CLE is imperative to comprhensive patient care.

\section{References}

1. Costner, MI and RD Sontheimer. Chapter 156: Lupus Erythematosus. Fitzpathrick's Dermatology in General Medicine, 7th ed. 2008. The McGraw-Hill Companies, Inc.

2. Blotzer J. Systemic Lupus Erythematosus I: historical aspects. Md State Med J 2007;100:896-898.

3. Patel P and Werth V. Cutaneous lupus erythematosus: a review. Dermatol Clin 2002; 20:373-385.

4. Werth VP. Clinical manifestation of cutaneous lupus erythematosus. Autoimmun Rev 2005;4:296-302.

5. Tebbe B and Orfanos C. Epidemiology and socioeconomic impact of skin disease in lupus erythematosus. Lupus 1997;6:96-104.

6. Durosaro $\mathrm{O}$ et al. Incidence of cutaneous lupus erythematosus, 1965-2005: a population-based study. Arch Dermatol 2009;145:249-253.

7. Osbermoser et al. Overview of common, rare and atypical manifestations of cutaneous lupus erythematosus and histopathological correlates. Lupus 2010; 19:1050-1070.

8. Fabbri et al. Cutaneous lupus erythematosus: diagnosis and management. Am J Clin Dermatol 2003;4:449-465.

9. Kuhn et al. Pathophysiology of cutaneous lupus erythematosus - novel aspects. Rheumatology. 2006;45:iii14-iii16.

10. Lin et al. Pathophysiology of cutaneous lupus erythematosus. Clinic Rev Allerg Immunol 2007;33:85-106.

11. Moghadam-Kia S et al. Cross-sectional analysis of a collaborative Web-based database for lupus erythematosus-associated skin lesions: prospective enrollment of 114 patients. Arch Dermatol 2009; 145:255-260.

12. Lee $\mathrm{H}$ and Sinha A. Cutaneous lupus erythematosus: Understanding of clinical features, genetic basis, and pathobiology of disease guides therapeutic strategies. Autoimmunity 2006; 39(6):433-444.

13. Zecevic et al. Skin lesions - an indicator of disease activity in systemic lupus erythematosus? Lupus 2001;10:364-367.

14. Yell JA, Mbuagbaw J, Burge SM. Cutaneous manifestations of systemic lupus erythematosus. Br J Dermatol 1996;135(3):355362.

15. Sontheimer RD, Henderson CL, Grau RH. Drug- induced subacute cutaneous lupus erythematosus: A paradigm for bedside-to-bench patient-oriented translational clinical investigation. Arch Dermatol Res 2009;301(1):65-70.

16. Parodi A et al. Measuring the activity of the disease in patients with cutaneous lupus erythematosus. Br J Dermatol 2000;142(3):457-460.

17. Zecevic RD et al. Skin lesions-an indicator of disease activity in systemic lupus erythematosus? Lupus 2001;10:364-367.

18. Camacho, IV. Acute cutaneous lupus erythematosus. Medscape. Updated 2011. Accessed online: http://emedicine.medscape. com/article/1065292-overview

19. Reich et al. The lupus band test in systemic lupus erythematosus patients. Therapeutics and Clinical Risk Management 2011;7:27-32.

20. Rothfield et al. Lupus erythematosus: cutaneous and systemic manifestations. Clinics in Dermatology 2006;24:348-362.

21. Werth, VP. Current treatment of cutaneous lupus erythematosus. Dermatology Online Journal 2001;7:2.

22. Wozniacka, A. and DP McCauliffe. Optimal use of antimalarials in treating cutaneous lupus erythematosus. Am J Clin Dermatol 2006;6:1-11.

23. Wallace DJ. Antimalarials: the "real" advance in lupus. Lupus 2001;10:385-387. 
24. Verny C et al. Heart conduction disorders in long term treatment with chloroquine: two new cases. Presse Med 1992;21:800-804.

25. Jewell ML, McCauliffe DP. Patients with cutaneous lupus erythematosus who smoke are less responsive to antimalarial treatment. J Am Acad Dermatol 2000;42:983-987.

26. Khamashta MA, Buchanan NM, Hughes GR. The use of hydroxychloroquine in lupus pregnancy: the British experience. Lupus 1996;5:S65-S66.

27. Levy RA et al. Hydroxychloroquine (HCQ) in lupus pregnancy: double blind and placebo controlled study. Lupus 2001;10:401404.

28. Costedoat-Chalumeau, $\mathrm{N}$ et al. Safety of hydroxychloroquine in pregnant patients with connective tissue diseases: a study of one hundred thirty-three cases compared with control group. Arthritis Rheum 2004;50:3056-3057.

29. Hall RP et al. Bullous eruption of systemic lupus erythematosus: dramatic response to dapsone therapy. Ann Intern Med 1982;97:165-170.

30. Tsutsui $\mathrm{K}$ et al. Widespread pruritic plaques in a patient with subacute cutaneous lupus erythematosus and hypocomplementemia: response to dapsone therapy. J Am Acad Dermtol 1996;35:313-315.

31. Neri R. et al. A case of SLE with acute, subacute and chronic cutaneous lesions successfully treated with Dapsone. Lupus 1999;8:240-243.

32. Shornick JK, Formica N, Parke AL. Isotretinoin for refractory lupus erythematosus. J Am Acad Dermatol 1991;24:49-52.

33. Pelle, MT and VP Werth. Thalidomide in cutaneous lupus erythematosus. Am J Clin Dermatol 2003;4:379-387.

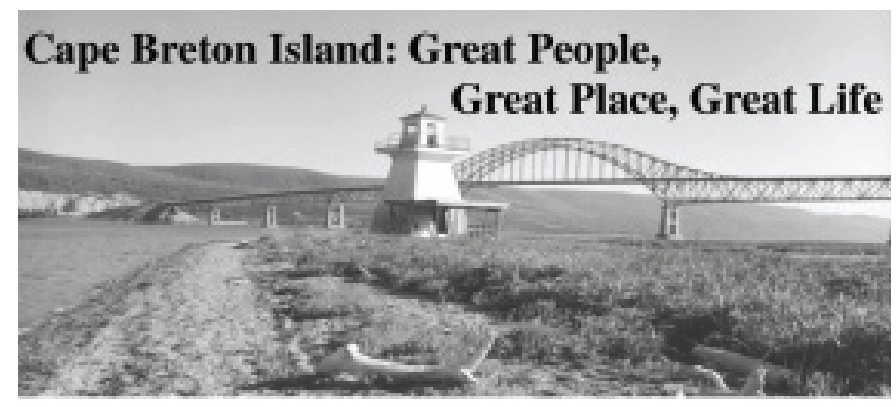

The Cape Breton District Health Authority has openings available in:
-Emergency Medicine

-Obstetrics/Gynecology
- Family Medicine -Urology
The District serves more than 130,000 people through regional, community and rural hospitals, Continuing Care, Mental Health Services, Addiction Services, Public Health Services and the Cape Breton Cancer Centre. Cape Breton's unique beauty, diverse culture and year-round recreational opportunities make the Island a vibrant place to work and play.

All candidates must be certified by the Royal College of Physicians and Surgeons or the Canadian College of Family Physicians or equivalent and be eligible for licensure in the provinee of Nova Scotia.

Inquiries and applications to:

Vice President, Medicine

Cape Breton District Health Authority

1482 George Street

Sydney, Nova Scotia BIP 1P3

Fax: (902) 567-7255

E-mail: jacksons@cbdha.nshealth_ca

Website: www.cbdha.nshealth.ca
Gee Ruken District HEALTH AUTHORTY
34. Campar et al. Refractory disease in Systemic Lupus Erythematosus. Autoimmun Rev 2011;10:685-692.

35. Albrecht et al. The CLASI (cutaneous lupus erythematosus disease area and severity index): an outcome instrument for cutaneous lupus erythematosus. J Inv Derm 2005;125:889-894.

36. Albrecht, J. and VP Werth. Development of the CLASI as an outcome instrument for cutaneous lupus erythematosus. Derma Ther 2007;20:93-101.

37. Klein et al. Quality of life in cutaneous lupus erythematosus. J Am Acad Dermatol 2011;64(5):849-858.

38. Callen, JP. Clinically relevant information about cutaneous lupus erythematosus. Arch Dermatol 2009; 145(3):316-319.

39. Tebbe B et al. Markers in cutaneous lupus erythematosus incdicating systemic involvement. A multienter study on 296 patients. Acta Derm Venerol 1997;77(4):305-308.

40. Tebbe B. Clinical course and prognosis of cutaneous lupus erythematosus. Clinics in dermatology 2004;22:121-124.

41. Shakra et al. Systemic lupus erythematosus and cancer: associated or not? Lupus 2002;11:137-144.

42. Grimaldo-Carjevschi et al. Squamous cell carcinoma on the palate in a patient with systemic lupus erythematosus: case report and review of literature. Lupus 2011;20:519-522.

\section{Live, work and play on the South Shore}

Imagine yourself kayaking, canocing, sailing, whale watching, diving or enjoying one of our many golf courses, hiking trails, theatres, galleries or museums. We are located on Nova Sontia's South Shore fone hour from metro Halifax), where miles of coastline are marked by beatiful, sandy beaches and picturesque towns including Bridgewacer, Chester, Liverpool, Luncnburg and Mahooe Bay; where hundreds of lakes, streams and rivers flow through small fishing villages and dense forests.

The District requires physicians for:

- Family Medicine (locations include Bridgewater, Caledonia, Chester, Lunenburg and Mahone Bay)

- Emergency Medicine

- Psychiatry - Bridgewater

- Walk-in Clinic-Bridgewater

- Locum opportunities available

Candidates must be eligible for licensure in Nova Scotia.

Inquiries and applications may be directed to:

Dr. Peter Vaughan, VP Medicine South Shore District Health Authority 90 Glen Allan Drive

Bridgewater NS B4V 3S6

Canada

Telephone: 902-527-5057

Fax: 902-527-5269

E-mail: pvaughanassdha.nshealth.ca

Website: www.southshorehealth.ca 